\title{
A LINEAR TRANSFORMATION WHOSE VARIABLES AND COEFFICIENTS ARE SETS OF POINTS
}

\author{
S. T. SANDERS, JR.
}

Introduction. While the theory of the linear transformation has been developed in great detail, attention has seldom ${ }^{1}$ been called to the transformation $T$ in which variables and coefficients are sets of points. Doubtless the nonexistence of a unique inverse transformation has occasioned this neglect. In this paper the writer studies the iteration of $T$.

Consider first the transformation

$$
T: \begin{aligned}
& x_{1}=a_{11} x_{1}^{\prime}+a_{12} x_{2}^{\prime} \\
& x_{2}=a_{21} x_{1}^{\prime}+a_{22} x_{2}^{\prime}
\end{aligned},
$$

whose set matrix is

$$
M=\left\|\begin{array}{ll}
a_{11} & a_{12} \\
a_{21} & a_{22}
\end{array}\right\|,
$$

where the $a$ 's and $x$ 's are sets of points, and the indicated sums and products refer to set operations. Applying $T$ to the primed variables, we have the product transformation

$$
T^{2}: \begin{aligned}
& x_{1}=a_{11}^{(2)} x_{1}^{\prime \prime}+a_{12}^{(2)} x_{2}^{\prime \prime} \\
& x_{2}=a_{21}^{(2)} x_{1}^{\prime \prime}+a_{22}^{(2)} x_{2}^{\prime \prime}
\end{aligned},
$$

of set matrix

$$
M^{2}=\left\|\begin{array}{cc}
a_{11}^{(2)} & a_{12}^{(2)} \\
a_{21}^{(2)} & a_{22}^{(2)}
\end{array}\right\|,
$$

where

$$
\begin{array}{ll}
a_{11}^{(2)}=a_{11}+a_{12} a_{21}, & a_{12}^{(2)}=a_{11} a_{12}+a_{12} a_{22}, \\
a_{21}^{(2)}=a_{21} a_{11}+a_{22} a_{21}, & a_{22}^{(2)}=a_{21} a_{12}+a_{22} .
\end{array}
$$

Transforming in turn each new set of variables, we obtain product transformations $T^{3}, T^{4}, \cdots$, whose set matrices are $M^{3}, M^{4}, \cdots$.

Presented to the Society, December 30, 1941 under the title On powers of a matrix whose elements are sets of points; received by the editors August 25, 1941.

${ }^{1}$ Lowenheim, Über Transformationen im Gebietekalkül, Mathematische Annalen, vol. 73 (1913), pp. 245-272; Gebietsdetermination, Mathematische Annalen, vol.79 (1919), pp. 223-236. 
Set matrices $M\left(a_{i j}\right)$ and $M^{\prime}\left(a_{i j}^{\prime}\right)$ are defined to be equal if $a_{i j}=a_{i j}^{\prime}$, $i, j=1,2, \cdots, n$, while if $a_{i j} \subset a_{i j}^{\prime},{ }^{2}$ then we shall write $M \subset M^{\prime}$. A sequence $M_{k}, k=1,2, \cdots$, of set matrices is increasing or decreasing according as $M_{k} \subset M_{k+1}$, or $M_{k+1} \subset M_{k}$.

From (1) follow $a_{11} \subset a_{11}^{(2)}$ and $a_{22} \subset a_{22}^{(2)}$. The assumptions $a_{12} \subset a_{12}^{(2)}$ and $a_{21} \subset a_{21}^{(2)}$ are equivalent to

$$
a_{12}+a_{21} \subset a_{11}+a_{22}
$$

which is a necessary and sufficient condition that $M \subset M^{2}$. But from (1) we have $a_{12}^{(2)}+a_{21}^{(2)} \subset a_{11}^{(2)}+a_{22}^{(2)}$, whence by (2) the inclusion $M^{2} \subset M^{4}$, and the following theorem is established.

THEOREM 1. The sequences $M^{2 k}$ and $M^{2 k+1}, k=1,2, \cdots$, of second order set matrices are increasing.

From (1) follow also $a_{12}^{(2)} \subset a_{12}$ and $a_{21}^{(2)} \subset a_{21}$, while the assumptions $a_{11}^{(2)} \subset a_{11}$ and $a_{22}^{(2)} \subset a_{22}$ are equivalent to

$$
a_{12} a_{21} \subset a_{11} a_{22}
$$

a necessary and sufficient condition that $M^{2} \subset M$. From (1) comes $a_{12}^{(2)} a_{21}^{(2)} \subset a_{11}^{(2)} a_{22}^{(2)}$, whence by (3) the inclusion $M^{4} \subset M^{2}$, and the following theorem.

Theorem 2. The sequences $M^{2 k}$ and $M^{2 k+1}, k=1,2, \cdots$, of second order set matrices are decreasing.

This theorem follows at once.

THEOREM 3. Even powers of a second order transformation are identical; likewise odd powers beyond the first.

The general case. Consider the transformation

of set matrix

$$
T: \quad x_{i}=\sum_{j=1}^{n} a_{i j} x_{j}^{\prime}, \quad i=1,2, \cdots, n,
$$

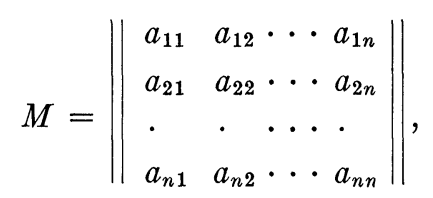

where the variables and coefficients are sets of points in a space whose generality is limited only by the following assumption.

\footnotetext{
${ }^{2}$ The symbol $\subset$ denotes inclusion.
} 
(A) The elements $a_{i j}$ of $M$ are independent point sets. That is, if products $P_{1}, P_{2}, \cdots, P_{k}, \cdots$ of elements of $M$ are such that

$$
P_{1} \subset \sum_{k \neq 1} P_{k}
$$

there is a subscript $k=k^{\prime}$ such that ${ }^{3} P_{1} \subset P_{k^{\prime}}$.

The following theorem will presently be established.

THEOREM 4. The iteration of the transformation $T$ of order $n$ leads to at most $(n-1)^{2}+N$ distinct transformations, where $N$ is the least common multiple of $1,2, \cdots, n$, and at most $N$ of these transformations recur periodically. If the coefficients of $T$ are independent, there are precisely $(n-1)^{2}+N$ disinct transformations of which $N$ recur periodically.

We shall denote by $a_{i j}^{(p)}$ the element in the $i$ th row and $j$ th column of $M^{p}$. Clearly $a_{i j}^{(p)}$ is a sum of products of the form

$$
P=a_{i k_{2}} a_{k_{2} k_{3}} a_{k_{3} k_{4}} \cdots a_{k p j},
$$

for all distinct ways of selecting the subscripts $k_{2}, k_{3}, \cdots, k_{p}$, from the integers $1,2, \cdots, n$. The characteristic interlocking form $k_{r} k_{s}$, $k_{s} k_{t}$ of the subscripts of consecutive factors of $P$ will be expressed in the word proper. Thus $a_{13} a_{32} a_{23} a_{32} a_{22}$ is a term of $a_{12}^{(5)}$ and a proper product, as opposed to the identical point set $a_{13} a_{32} a_{23} a_{22}$.

The order of a product $P$ is the number of factors occurring in the product. The orders of products $P, C, \cdots$, will be denoted by $p, c, \cdots$. A cycle of $P$ is a proper product $a_{k_{1} k_{2}} a_{k_{2} k_{3}} \cdots a_{k_{c} k_{1}}$ of factors of $P$ in which the subscripts $k_{1}, k_{2}, \cdots, k_{c}$ are distinct. It is convenient to denote such a cycle by $C_{k_{1}}$. Thus $a_{12} a_{23} a_{33} a_{32}$ involves the cycles $C_{2}=a_{23} a_{32}, C_{3}=a_{33}$, and $C_{3}^{\prime}=a_{32} a_{23}$. A closed cycle of $P$ is one whose factors occur consecutively in $P$. A product $P$ is $c$-cyclic if every product of $c$ consecutive factors is a closed cycle. It follows that these closed cycles are cyclic permutations of a single cycle $C$, known as the defining cycle of the product $P$. Thus $a_{23} a_{31} a_{12} a_{23} a_{31}$ is 3-cyclic, with the defining cycle $C_{2}=a_{23} a_{31} a_{12}$.

Proper products are coterminal if corresponding terminal subscripts are equal. Thus $P=a_{12} a_{23} a_{34}$ and $P_{1}=a_{15} a_{54}$ are coterminal. Such products clearly are terms of elements similarly situated in set matrices $M^{p}$ and $M^{p^{\prime}}$. It is convenient to denote a proper product $a_{i k_{2}} a_{k_{2} k_{3}} \cdots a_{k_{p} j}$ by $P_{i j}$. If coterminal proper products $P_{i j}, P_{i j}^{\prime}$ are

\footnotetext{
${ }^{3}$ Thus, under (A), such inclusions as $a_{12} a_{23} \subset P=a_{14}+a_{13} a_{32}$ cannot hold, since $P$ does not involve one of the terms $a_{12}, a_{23}, a_{12} a_{23}$.
} 
such that $p_{i j}^{\prime}<p_{i j}$ while $P_{i j} \subset P_{i j}^{\prime}$, we say that $P_{i j}^{\prime}$ is a contraction of $P_{i j}$, while $P_{i j}$ is an expansion of $P_{i j}^{\prime}$. Thus $a_{12} a_{23}$ is a contraction of $a_{12} a_{24} a_{42} a_{23}$, while the latter product is an expansion of the former.

The following lemmas are immediate consequences of the preceding definitions.

Lemma 1. A proper product of order exceeding $n-1$ involves a closed cycle.

Lemma 2. The deletion of a closed cycle from a proper product of greater order yields a contraction of the product.

LeMma 3. The insertion of a cycle $C_{k_{\mathrm{s}}}$ into a proper product immediately following a factor $a_{k_{r} k_{s}}$, or immediately preceding a factor $a_{k_{\mathbf{s}} k_{t}}$, yields an expansion of the product. Thus the appropriate insertion of $C_{1}=a_{14} a_{41}$ into $P_{12}=a_{13} a_{31} a_{12}$ yields $P_{12}^{\prime}=a_{13} a_{31} a_{14} a_{41} a_{12}$, or $a_{14} a_{41} a_{13} a_{31} a_{12}$.

Consider the sequence

$$
P_{i j}, P_{i j}^{\prime}, P_{i j}^{\prime \prime}, \cdots, P_{i j}^{(k)}
$$

in which each element after the first is obtained from its predecessor by the deletion of a closed cycle. The last element can involve no cycle, or is itself a cycle, and is called a stem of $P_{i j}$. The definition is not unique, since $P_{i j}^{(k)}$ clearly varies with the sequence of cycles of $P_{i j}$ whose deletion leads to (4). Thus $a_{12} a_{23} a_{32} a_{24} a_{43}$ has the stems $a_{12} a_{23}$ and $a_{12} a_{24} a_{43}$.

From Lemmas 1 and 2 follows this lemma.

Lemma 4. A stem of a proper product $P$ is a contraction of $P$ which has no contraction. The order of a stem cannot exceed $n$.

Increasing sequences. We first prove this lemma.

LEMMA 5. For every integer $c$ not exceeding p nor $n$, there occurs in $M^{p}$, $p \geqq 2$, an element involving a term which is a c-cyclic product.

If $p=m c$ each diagonal element $a_{i i}^{(p)}$ involves certain $c$-cyclic terms in which a cycle $C$ is repeated $m$ times. If $p=m c+r, 1 \leqq r<c$, each element $a_{i j}^{(p)}, i \neq j$, involves certain $c$-cyclic terms in which the $r$ th factor, and hence the $p$ th, is $a_{k j}$. Thus, for $n \geqq 4, a_{33}^{(4)}$ involves the 2-cyclic term $a_{31} a_{13} a_{31} a_{13}$, while $a_{24}^{(5)}$ involves the 3 -cyclic term $a_{21} a_{14} a_{42} a_{21} a_{14}$.

Theorem 5. Let $M$ be a set matrix of order $n$ whose elements are independent. The sequence $M^{p_{1}}, M^{p_{2}}, \cdots, M^{p_{r}}, M^{p_{r+1}}, \cdots, p_{r}<p_{r+1}$, is increasing if and only if $p_{1}>n-1$, while $p_{r+1}-p_{r}$ is a multiple of $1,2, \cdots, n$. 
By Lemma 1 a term $P$ of $a_{i j}^{\left(p_{r}\right)}$ involves a closed cycle $C$. Since $p_{r+1}-p_{r}$ is a multiple of $c$ we can insert appropriately into $P$ sufficient repetitions of $C$ to yield by Lemma 3 an expansion $\bar{P}=P$ of order $p_{r+1}$. Thus $\bar{P}$ is the required term of $a_{i j}^{\left(p_{r+1}\right)}$.

Conversely, if $p_{1} \leqq n-1$ there is a term $P^{\prime}$ of $a_{i j}^{\left(p_{1}\right)}$ involving no cycle, and which by Lemma 1 and (A) is not contained in $a_{i j}^{(p)}$ for $p=p_{2} \geqq n$. And if $p_{1}>n-1$ while $p_{r+1}-p_{r}$ is not a multiple of $c \leqq n$, there is by Lemma 5 an element $a_{i j}^{\left(p_{r}\right)}$ of $M^{p_{r}}$ involving a term $P_{r}$ which is a $c$-cyclic product. Now a product $P_{r+1}$ which contains $P_{r}$ can by (A) involve only factors of $P_{r}$, and is hence $c$-cyclic. But since $p_{r+1}-p_{r}$ is not a multiple of $c$, it follows that $P_{r+1}$ is not coterminal with $P_{r}$, and so is not a term of $a_{i j}^{\left(p_{r+1}\right)}$. From (A) we conclude that $P_{r}$ is not contained in the set $a_{i j}^{\left(p_{r+1}\right)}$ and the theorem is established.

Decreasing sequences. Before proceeding we prove two lemmas.

Lemma 6. Let $P$ be a proper product, $S$ a sequence of cycles of $P$ determining a stem $P^{\prime}, h=h(S)$ the highest common factor of the orders of the cycles of $S$, and c the greatest of these orders. There is a contraction $\bar{P}$ of $P$ involving every subscript of $P$, and such that $\bar{p} \leqq c(n-c+2)-1$, $p \equiv \bar{p}(\bmod h)$.

Consider the following sequence

$$
S^{\prime}: \quad P^{\prime}, C_{1}, C_{2}, \cdots, C_{k},
$$

in which: (i) $C_{1}$ is a cycle of $S$ involving a subscript not found in $P^{\prime}$, and has the maximum order of all such cycles. (ii) Each $C$ following $C_{1}$ is a cycle of $S$ involving a subscript which has previously appeared in $S^{\prime}$, and one which has not done so. Further, all such cycles of $S$ are in the sequence $S^{\prime}$.

It is easily shown that every subscript involved in $P$ occurs in some cycle of $S^{\prime}$. For let $k_{s}$ be the first subscript of $P$ not found in $S^{\prime}$. Since $k_{s}$ cannot occur in the stem $P^{\prime}$, it must first appear in $P$ in a factor of the form $a_{k_{r} k_{s}}$. But $k_{s}$ occurs in some cycle $C^{\prime}$ of $S$, while $k_{r}$ occurs in a cycle of $S^{\prime}$. We infer from (ii) that $C^{\prime}$ is a cycle of $S^{\prime}$, and a contradiction is reached.

Now if the cycles $C_{1}, C_{2}, \cdots, C_{k}$ exist, at least one involves a subscript of $P^{\prime}$; for the contrary assumption leads to a contradiction, as in the above argument, on consideration of the first appearance in $P$ of a subscript of $C_{1}, C_{2}, \cdots, C_{k}$. It follows that the cycles of $S^{\prime}$ can be combined with $P^{\prime}$ into a contraction $\bar{P}$ of $P$ which involves every subscript of $P$.

Consider now the order of $\bar{P}$. We have 


$$
\bar{p}=p^{\prime}+\sum_{t=1}^{k} c_{t},
$$

$c_{t} \leqq c$.

If $p^{\prime} \leqq c-2, P^{\prime}$ and $C_{1}$ together involve at least $c$ distinct subscripts,

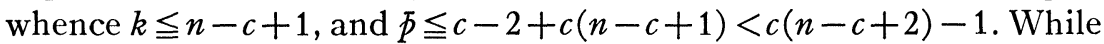
if $p^{\prime}>c-2, P^{\prime}$ and $C_{1}$ together involve at least $p^{\prime}+1$ distinct subscripts, whence $k \leqq n-p^{\prime}$, and $p \leqq p^{\prime}+c\left(n-p^{\prime}\right)=c n-p^{\prime}(c-1) \leqq c n$ $-(c-1)^{2}=c(n-c+2)-1$.

The congruence $p \equiv \bar{P}(\bmod h)$ follows from the definition of $P^{\prime}$ and $\bar{P}$. The lemma is established.

LeMma 7. Let $c_{1}>c_{2}>\cdots>c_{m}$ be a set $S_{1}$ of positive integers whose highest common factor is $h=h\left(S_{1}\right)$. The equation $c_{1} x_{1}+c_{2} x_{2}+\cdots+c_{m} x_{m}$ $=k$ has a non-negative integral solution $\left(x_{1}^{\prime}, x_{2}^{\prime}, \cdots, x_{m}^{\prime}\right)$ for every integer $k$ which is a multiple of $h$ exceeding $\left(c_{1} c_{2} / h\right)-c_{1}-c_{2}{ }^{4}$

THEOREM 6. Let $M$ be a set matrix of order $n$ whose elements are independent. The sequence $M^{p_{1}}, M^{p_{2}}, \cdots, M^{p_{r}}, M^{p_{r+1}}, \cdots, p_{r}<p_{r+1}$, is decreasing if and only if $p_{1}>(n-1)^{2}$, while $p_{r+1}-p_{r}$ is a multiple of $1,2, \cdots, n$.

Sufficiency. For any term $P$ of $a_{i j}^{\left(p_{r+1}\right)}$; the theorem asserts the existence of a term $P_{1}$ of $a_{i j}^{\left(p_{r}\right)}$ such that $P \subset P_{1}$. Our procedure is to insert into a contraction of $P$ appropriate cycles of $P$ of the precise total order required to yield the desired product $P_{1}$.

Let $P^{\prime}$ be a stem of $P$, determined by a sequence $S$ of cycles of $P$. Let $\bar{P}$ be the contraction of $P$ presented in Lemma 6; and let $S_{1}: C_{1}, C_{2}, \cdots, C_{m}$, be cycles of $S$ among whose orders, $c_{1}>c_{2}>\cdots>c_{m}$ occur all orders of cycles of $S$. If $m=1$, the required term $P_{1}$ is clearly encountered in the sequence (4) of products defining $P^{\prime}$.

Case 1. $c_{1}<n$.

By Lemma 6 we have

$$
\begin{aligned}
p_{r}-\bar{p}>(n-1)^{2} & -c_{1}\left(n-c_{1}+2\right)+1 \\
& =c_{1}\left(c_{1}-1\right)-c_{1}-\left(c_{1}-1\right)+(n-1)\left(n-c_{1}-1\right),
\end{aligned}
$$

whence

$$
p_{r}-\bar{p}>c_{1}\left(c_{1}-1\right)-c_{1}-\left(c_{1}-1\right) \geqq \frac{c_{1} c_{2}}{h}-c_{1}-c_{2} .
$$

Now by the same lemma, $p_{r+1}-\bar{p} \equiv 0(\bmod h)$, hence from $p_{r+1}-p_{r} \equiv 0$ $(\bmod h)$ follows

4 This lemma is readily established by mathematical induction. However, a better bound on $k$, namely, $\left(c_{1} c_{n} / h\right)-c_{1}-c_{m}$ has been communicated to the author by Dr. Alfred Brauer. 


$$
p_{r}-\bar{p} \equiv 0(\bmod h) \text {. }
$$

By (5), (6), and Lemma 7 there is established the existence of nonnegative integers, $x_{1}^{\prime}, x_{2}^{\prime}, \ldots, x_{m}^{\prime}$ such that

$$
\bar{p}+\sum_{i=1}^{m} c_{t} x_{t}^{\prime}=p_{r}
$$

By Lemmas 6 and 3 we can insert the cycles $C_{1}, C_{2}, \cdots, C_{m}$ into $\bar{P}$ and obtain the required product $P_{1}$.

Case 2. $c_{1}=n, c_{2}<n-1$.

Again by Lemma 6

$$
\begin{aligned}
p_{r}-p>(n-1)^{2}- & c_{1}\left(n-c_{1}+2\right)+1 \\
& =c_{1}\left(c_{1}-2\right)-c_{1}-\left(c_{1}-2\right)+(n-2)\left(n-c_{1}\right),
\end{aligned}
$$

whence

$$
p_{r}-p>c_{1}\left(c_{1}-2\right)-c_{1}-\left(c_{1}-2\right) \geqq \frac{c_{1} c_{2}}{h}-c_{1}-c_{2},
$$

and the proof proceeds as in Case 1.

Case 3. $c_{1}=n, c_{2}=n-1$.

Here the contraction $P^{\prime}$, instead of $\bar{P}$, is employed. We have by Lemma 4

$$
p_{r}-p^{\prime}>(n-1)^{2}-n=c_{1} c_{2}-c_{1}-c_{2} .
$$

Since $h=1$ it follows by Lemma 7 that non-negative integers, $x_{1}^{\prime}, x_{2}^{\prime}$, exist such that $p^{\prime}+c_{1} x_{1}^{\prime}+c_{2} x_{2}^{\prime}=p_{r}$. Thus since $P^{\prime}$ must involve a subscript of $C_{1}$ and $C_{2}$, it is possible to insert the cycles $C_{1}, C_{2}$, into $P^{\prime}$ and obtain by Lemma 3 the required product $P_{1}$.

Necessity. As in Theorem 5 it can be shown that $p_{r+1}-p_{r}$ must be a multiple of $1,2, \cdots, n$; while for the condition $p_{1}>(n-1)^{2}$, it will suffice to produce a term of an element of $M^{p_{2}}$ which is not contained in the corresponding element of $M^{p_{1}}$, although $p_{2}-p_{1}$ is a multiple of $1,2, \cdots, n$.

Consider the $n$-cyclic product $P_{1}^{\prime}$ of order $p_{1}+n-1$ whose defining cycle is $C_{1}=a_{12} a_{23} \cdots a_{n 1}$, and the $(n-1)$-cyclic product $P_{2}^{\prime}$ of order $p_{2}-p_{1}-n+1$ whose defining cycle is $C_{2}=a_{23} a_{34} \cdots a_{n 2}$. By inserting $P_{2}^{\prime}$ into $P_{1}^{\prime}$ following any factor $a_{12}$, a proper product $P$ of order $p_{2}$ is obtained which is a term of an element of the first row of $M^{p 2}$. Now from (A) and the structure of $P$ it follows that any proper coterminal product containing $P$ can be had from $P$ by deletion of the cycles $C_{1}, C_{2}$. We are thus led to the equation

$$
n x_{1}+(n-1) x_{2}=p_{2}-p_{1},
$$


with the restrictions

$$
\begin{aligned}
n x_{1} & \leqq p_{1}+n-1 \\
(n-1) x_{2} & \leqq p_{2}-p_{1}-n+1
\end{aligned}
$$

By (7) and (9), $x_{1}$ is a positive multiple of $n-1$, while from $p_{1} \leqq(n-1)^{2}$ we have by (8), $n x_{1} \leqq n(n-1)$. Thus $x_{1}=n-1$, but it is clear that the deletion of $n-1$ cycles $C_{1}$ from $P$ will yield a product ${ }^{5}$ whose first factor is $a_{23}$. From (A) it follows that $P$ is not contained in the corresponding element of $M^{p_{1}}$, and the theorem is established.

Equality of matrices. Theorems 5 and 6 provide conditions for increase and decrease, respectively, in a sequence of ascending powers of $M$. In summation we have the following theorem:

THEOREM 7. Let $M$ be a set matrix of order $n$ whose elements are independent. The equality $M^{p_{1}}=M^{p_{2}}, p_{2}>p_{1}$, holds if and only if $p_{1}>(n-1)^{2}$, while $p_{2}-p_{1}$ is a multiple of $1,2, \cdots, n$.

Theorem 4 is an immediate consequence.

Southwestern Louisiana Institute

5 Thus for $n=3, p_{1}=4, p_{2}=10$, we have $P=a_{12} a_{23} a_{32} a_{23} a_{32} a_{2 \mathrm{~s}} a_{31} a_{12} a_{23} a_{31}$ which has no contraction of order 4 . 